\title{
Presencia de Plasmodium brasilianum (Apicomplexa, Plasmodidae) en el mono congo (Alouatta palliata, Primates: Cebidae) de Costa Rica. Importancia epidemiológica en relación con el ser humano
}

\author{
MISAEL CHINCHILLA****, OLGA MARTA GUERRERO*, \\ GUSTAVO GUTIÉRREZ** y RONALD SÁNCHEZ*****
}

\begin{abstract}
Plasmodium brasilianum (APICOMPLEXA, PLASMODIDAE) IN THE CONGO MONKEY Alouatta brasilianum (PRIMATE: CEBIDAE) OF COSTA RICA. EPIDEMIOLOGICAL IMPORTANCE RELATED TO HUMAN
\end{abstract}

Plasmodium brasilianum, a malaria parasite of Primates, was found for the first time in Costa Rica in 6 of 104 howler monkeys (Alouatta palliata) studied. Animals were captured and anaesthesied with dards containing equal parts of tiletamine hidrochloride and Zolazepam (Zoletil®). To study blood parasites, smears were prepared, fixed in methyl alcohol and stained by Giemsa technique. Young and advanced trofozoites (band forms) as well as gametocytes and squizonts ("rosseta" forms) were found. Since this parasite is very similar to $\boldsymbol{P}$. malariae, a human parasite that has been found in Costa Rica, the presence of $\boldsymbol{P}$. brasilianum is discussed on the epidemiological point of view.

Key words: Apicomplexa, Plasmodium brasilianum, Plasmodium malariae, Alouatta palliata, howler monkey.

\section{INTRODUCCIÓN}

Dentro de las especies del género Plasmodium, parásitas de primates no humanos a saber: $P$. brasilianum, $P$. coatneyi, $P$. cinomolgi, $P$. fieldi, $P$. fragile, $P$. hylobati, $P$. gonderi, $P$. inui, $P$. knowlesi, $P$. simium y P.reichenowi ${ }^{1}$, el primero ha sido confirmado también en el ser humano² de ahí su importancia epidemiológica.

Después de que Gonder \& Berenberg-Gossler (1908) encontraron este parásito en la sangre de un mono Brachyurus calvus importado de la región amazónica de Brasil, se ha conocido su presencia en Panamá, Brasil, Venezuela, Colombia, Perú y Guayana Francesa tal como lo cita Coatney et $\mathrm{al}^{3}$. Este parásito ha sido

\footnotetext{
* Centro de Investigación en Enfermedades Tropicales, Departamento de Parasitología, Facultad de Microbiología, Universidad de Costa Rica.

** Escuela de Biología, Universidad de Costa Rica.

*** Departamento de Investigación, Universidad de Ciencias Médicas (UCIMED).

**** Sede de Occidente, Universidad de Costa Rica.

Dirección: Misael Chinchilla Carmona Ph.D. Departamento de de Parasitología, Facultad de Microbiología, Universidad de Costa Rica. San José, Costa Rica, América Central. Email: chinchillacm@UCIMED.com
} 
encontrado en primates de los géneros: Alouatta, Ateles, Brachyteles, Brachyurus, Callicebus, Cebus, Chiropotes, Lagothryx y Saimiri ${ }^{3,4}$, así como en los géneros Saguinus y Pithecia ${ }^{5}$ y Aotus ${ }^{4}$.

Este organismo, al igual que todas las especies del género Plasmodium, es diheteroxeno, llevando a cabo su ciclo de vida entre los primates en donde se desarrolla fundamentalmente la fase asexual y mosquitos del género Anopheles, en donde se realiza la fase sexual. Sus vectores conocidos conocidas son: A. tarsimaculatus (= aguasalis) y A. albimanus $^{3}$, pero también se han considerado a las especies $A$. mediopunctaus, $A$. nuneztovari, A. oswaldoi, A. triannulatus y $A$. shannoni como los posibles vectores del parásito en el Amazonas brasileño ${ }^{6}$. Infecciones experimentales se han logrado en A. aztecus, A. atroparvus $^{7}$ y también en A. freeborni, A. dirus, A. maculatus y A. gambiae ${ }^{3}$.

Al igual que en $P$. knowlesi y en $P$. simium, especies en que se han reportado infecciones naturales o experimentales en el ser humano, en $P$. brasilianum también se ha observado lo mismo $^{8-10}$. En realidad esta especie ha sido relacionada estrechamente con $P$. malariae, parásito humano, tanto que algunos los consideran a ambos, desde el punto de vista genético, inmunológico y epidemiológico, como una sola especie ${ }^{5,11-13}$.

Este trabajo pretende, no sólo informar de la presencia de este parásito por primera vez en primates de la especie Alouatta palliata en Costa Rica, sino también realizar una breve revisión sobre los aspectos más relevantes del mismo, especialmente en cuanto a su relación con $P$. malariae y la posibilidad de futuras infecciones para el ser humano.

\section{MATERIAL Y MÉTODOS}

Dado que este trabajo es parte de un gran proyecto integral en que se estudian muchos aspectos relacionados con la biología de los primates en Costa Rica, existen ya varias publicaciones en que se describen los métodos generales ${ }^{14,15}$. Brevemente, 104 ejemplares de monos congo (A. palliata) provenientes de varias zonas del país (San Ramón de Alajuela; Chomes, Parque Manuel Antonio, Isla Chira y Quepos (La Merced, La Uvita) de Puntarenas; Parque Palo Verde, Gran Nicoya, Playa Potrero de
Guanacaste; Cahuita de Limón. Los animales fueron capturados con dardos conteniendo compuestos anestésicos aplicados mediante técnicas conocidas ${ }^{16,17}$. El anestésico usado fue el hidrocloruro de tiletamina, un análogo de ketamina, combinado con zolazepam (una pirazolodiazepina) conocido como Zoletil®, en una combicación 1:1. Los primates fueron recibidos al caer en redes sostenidas por 4 personas. Se practicaron varios exámenes pero específicamente en cuanto a la obtención de la muestra de los animales para el estudio de parásitos sanguíneos, la sangre no sólo se extrajo en tubos con anticoagulante para la preparación de cultivos, sino que se prepararon extendidos en portaobjetos para luego fijarlos en alcohol metílico y teñirlos con el colorante de Giemsa. Estos extendidos fueron estudiados en el laboratorio para determinar morfológicamente los diferentes estados evolutivos del protozoo a saber: trofozoitos, gametocitos y esquizontes en diferentes estados de desarrollo del ciclo evolutivo del género Plasmodium.

Para documentar el informe se midieron esos estados evolutivos y se tomaron las correspondientes fotografías, realizando un análisis morfológico comparativo especialmente entre $P$. brasilianum, $P$. malariae y $P$. knowlesi que son las tres especies mas similares.

\section{RESULTADOS}

De los 104 animales estudiados se encontraron 3 ejemplares provenientes de Cahuita, Limón que presentaban formas muy inmaduras del parásito. Además en la zona de Quepos (La Uvita), 3 de los primates ahí estudiados resultaron positivos con una regular cantidad de formas de trofozoitos, gametocitos y esquizontes fácilmente identificables y apropiados para realizar la clasificación correspondiente. La Figura 1 presenta una composición de los diversos estados evolutivos del protozoo encontrado en la sangre de nuestros monos congo o aulladores, comenzando con el trofozoito joven (Figura 1 a). Obsérvese también las formas típicas en banda de los trofozoitos más avanzados (Figuras $1 \mathrm{~b}$, c), así como los esquizontes con la disposición característica de 8 o menos merozoitos alrededor del cuerpo residual, por lo que se les ha designado con el nombre de forma en margarita o "rosseta" en italiano (Figuras $1 \mathrm{~d}$, e). Nótese además las 


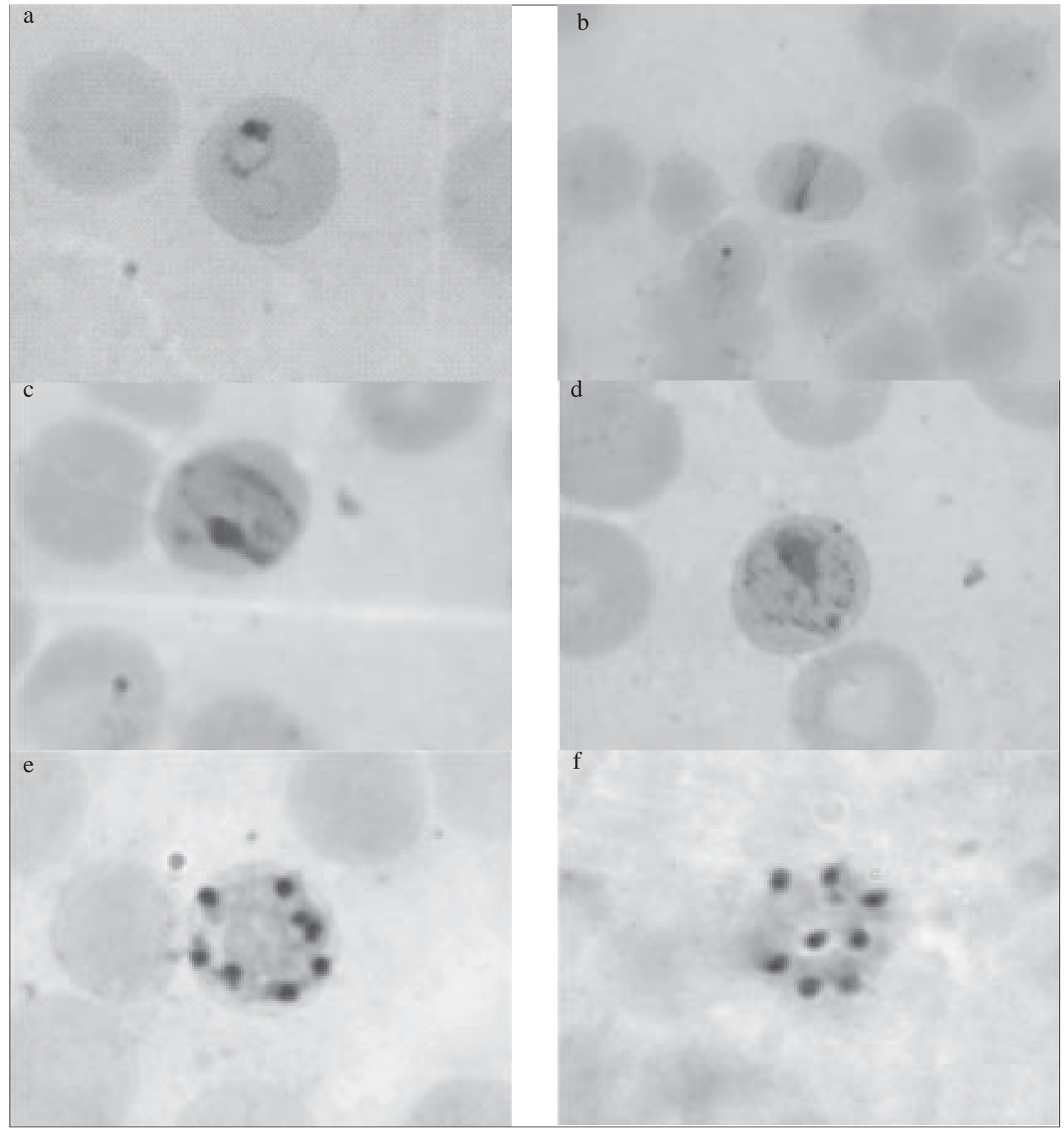

Figura 1. Estados evolutivos de Plasmodium brasilianum encontrados en sangre periférica de primates de la especie Alouatta palliata. a. Trofozoito joven, b. Trofozoito joven en banda, c. Trofozoito avanzado en banda, d. Gametocito, e. Esquizonte, f. Esquizonte.

formas redondeadas de los gametocitos (Figura $1 \mathrm{f}$ ).

\section{DISCUSIÓN}

La ratificación de que el parásito encontrado es $P$. brasilianum se basa en las características morfológicas de los estados evolutivos encontrados en la sangre de los monos, especialmente de las llamadas formas en banda características de los trofozoitos avanzados (Figuras 1 b, c). También tiene carácter diagnóstico la morfología de los esquizontes en 
cuanto a la disposición periférica de los merozoitos (Figuras $1 \mathrm{~d}$, e). Estas características sirven para diferenciarlo de las otras especies de Plasmodium presentes en monos del género Alouatta.

El hecho de que $P$. brasilianum es prácticamente indiferenciable morfológicamente de $P$. malariae ${ }^{5}$, un parásito causante de la llamada fiebre cuartana en el hombre, nos inclina a pensar, de acuerdo con nuestros hallazgos, que en cierto modo los monos aulladores de nuestro país pueden considerarse como verdaderos reservorios de agentes de la malaria de seres humanos. Llama la atención que los casos humanos bien documentados por $P$. malariae en Costa Rica ${ }^{18}$ son pocos. En efecto es $P$. vivax la especie predominante siguiéndole las infecciones por $P$. falciparum. Sin embargo el hecho de que $P$. malariae, al igual que el $P$. brasilianum, parasitan únicamente los glóbulos rojos más viejos, hace que que la sintomatología causada por estos parásitos sea bastante benigna, lo cual podría ser la causa de que se haya confundido con otras entidades nosológicas con síntomas tan comunes como la fiebre y el malestar general, también presentes en la malaria.

De todas maneras, resulta interesante establecer que en nuestro país, los monos de las dos zonas costeras son potenciales reservorios de al menos una especie productora de malaria, la cual eventualmente podría desarrollarse o ha venido infectando silenciosamente al ser humano. Esta idea se apoya precisamente en la similitud extraordinaria entre $P$. malariae y $P$. brasilianum mencionada y demostrada en varios estudios $^{5,11-13}$.

Queda por determinar la presencia de los trasmisores de este parásito en las áreas estudiadas, lo cual es muy probable que ocurra, dada la cantidad de mosquitos ahí presentes, dentro de los que el género Anopheles, trasmisor por excelencia de los agentes etiológicos de la malaria, es relativamente frecuente.

Con este hallazgo se agrega a los parásitos intestinales previamente comunicados ${ }^{14} \mathrm{y}$ al informe de la presencia de Trypanosoma minasense ${ }^{15}$, el nombre de un parásito más lo que enriquece el conocimiento de la fauna parasitológica de los monos de la especie $A$. palliata estudiados prácticamente en todo el país.

\section{RESUMEN}

La especie productora de malaria en primates, Plasmodium brasilianum, fue encontrada por primera vez en Costa Rica en 6 de 104 ejemplares de monos congo o aulladores (Alouatta palliata). Los animales fueron capturados y anestesiados por medio de dardos que contenían hidrocloruro de tiletamina y zolazepam (Zoletil®) combinados en partes iguales. Para estudiar estos animales por parásitos sanguíneos, se prepararon frotis sanguíneos que luego se tiñeron y se estudiaron en el laboratorio, encontrándose las formas de trofozoitos jóvenes o avanzados así como gametocitos y esquizontes. La morfología característica de algunos estados evolutivos, como por ejemplo, las formas en banda de trofozoitos avanzados y los esquizontes en forma de margarita o "rosetta" permitieron el diagnóstico de la especie. Puesto que se han encontrado casos humanos infectados con este organismo y éste es casi indiferenciable de Plasmodium malariae, una especie parásita del ser humano, se discute el hallazgo de este parásito desde un punto de vista epidemiológico en el área de la salud.

\section{REFERENCIAS}

1.- LECLERC M C, HUGOT J P, DURAND P, RENAUD F. Evolutionary relationships between 15 Plasmodium species from New and Old World primates (including humans): a $18 \mathrm{~S}$ rDNA cladistic analysis. Parasitology 2004; 129: 1-8.

2.- LOURENCO-DE-OLIVEIRA R, DEANE L M. Simian malaria at sites in the brazilian amazon. I-The infection rates of Plasmodium brasilianum in non-human primates. Mem Inst Oswaldo Cruz, Rio de Janeiro 1995; 90: 331-9.

3.- CD-ROOM. The primate malarias (Original book published 1971). Coatney G R, Collins M E, Warren M, Contacos P G. División of Parasitic Disease, producers. Versión 1.0. Atlanta, GA: CDC; 2003.

4.- COGSWELL F B. Malaria and piroplasms of nonhuman primates. Companion and Exotic Animal Parasitology, Browman D.D (Ed.). International Veterinary Information Service 2000; (www.ivis.org).

5.- FANDEUR T, VOLNEY B, PENEAU C, DE THOISY B. Monkeys of the rainforest in French Guiana are natural reservoirs $P$. brasilianum/P. malariae malaria. Parasitology 2000; 120: 11-21.

6.- LOURENCO-DE-OLIVEIRA R, LUZ L B. Simian malaria at two sites in the brazilian Amazon II. Vertical distribution and frequency of anopheline species inside and outside the forest. Mem Inst Oswaldo Cruz, Rio de Janeiro 1996; 91: 687-94.

7.- GARNHAM P C C, BAKER J R, NESBITT P E. 
Trasmission of Plasmodium brasilianum by sporozoites and the discovery of an exoerythrocytic schizont in the monkey liver. Parasitología 1963; 5: 5-9.

8.- DEANE L M, DEANE M P, FEREIRA NETO J. Studies on transmission of simian malaria and on a natural infection of man with Plasmodium simium in Brazil. Bull World Health Organ 1966; 35: 805-8.

9.- JONGWUTIWES S, PUTAPORNTIP C, IWASAKI T, SATA T, KANBARA H. Naturally acquired Plasmodium knowlesi malaria in human, Thailand. Emerg Inf Dis 2004; 10: 2211-3.

10.- COLLINS W E. CDC Program Reactivates Archived Parasites. Newsletter of the Malaria Research and Reference Reagent Resource Center 2003; www.malaria.mr4.org malaria@atcc.org

11.- CHIZZOLINI C, SULZER A J, OLSEN-RASMUSSEN M A, COLLINS W E. Epstein-Barr Virus transformation of Saimiri sciureus (squirrel monkey) B cells and generation of a Plasmodium brasilianum specific monoclonal antibody in $P$. brasilianuminfected monkeys. Infec. Immun 1991; 59: 2285-9.

12.- GALLAND G G. Role of the squirrel monkey in biomedical and behavioral Research. ILAR Journal The squirrel monkey in biomedical and behavioral research 2000; 41: 1-10.

13.- CURADO I, DUARTE A M, LAL A A, OLIVEIRA S G, KLOETZEL J K. Antibodies anti Bloodstream and Circumsporozoite Antigens (Plasmodium vivax and Plasmodium malariae/P. brasilianum) in Areas of Very Low Malaria Endemicity in Brazil. Mem Inst Oswaldo
Cruz 1997; 92: 235-43.

14.- CHINCHILLA M, GUERRERO O. M, GUTIÉRREZ G, SÁNCHEZ R, RODRÍGUEZ B. Parásitos intestinales en monos congo Alouatta palliata (Primates: Cebidae) de Costa Rica. Rev Biol Trop 2005; 53: 437-45.

15.- CHINCHILLA M, TROYO A, GUTIÉRREZ G, SÁNCHEZ R. Presencia de Trypanosoma minasense (Kinetoplastida: Trypanosomatidae) en Alouatta palliata (Primates: Cebidae) de Costa Rica. Parasitol Latinoam 2005; 60: 90-92.

16.- GLANDER K E, FEDIGAN L M, FEDIGAN L, CHAPMAN C. Field Methods for Capture and Measurement of three monkey species in Costa Rica. Folia Primatologica 1991; 57: 70-82.

17.- SWAM G E. A summary of the practical aspects of drugs commonly used for the restraint of wild animals. Capture and Care Manual online 1992; http:// www.wildlifedecisionsupport.com/captureandcare/ sectiona/drugs/01_swan.htm

18.- VARGAS M. Diagnóstico situacional de la malaria y el uso de DDT en Costa Rica. Organización Panamericana de la Salud/Organización Mundial de la Salud (OPS/OMS) 2001; 217 p.

Agradecimientos: Este trabajo fue financiado en parte por la Vicerrectoría de Investigación de la Universidad de Costa Rica a través de los proyectos VI-III-A1-015 y 803-A1-745 y el Departamento de Investigación de la Universidad de Ciencias Médicas. 